\title{
DYNAMICS OF AFFECTIVE HABITUATION TO TOUCH DIFFER ON THE GROUP AND INDIVIDUAL LEVEL
}

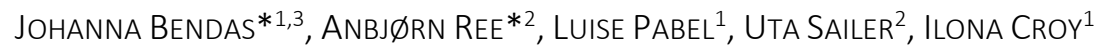

1 Department of Psychotherapy and Psychosomatics, Medical Faculty, TeChnische Universität Dresden, GERMANY

2 Institute of Basic Medical SCIEnCES, Department Of BehaVIOURAL Medicine, FACUlty of MediCine, University OF OSLO, NORWAY

3 Department of Pediatrics, Ev.-Luth. Diakonissenanstalt Zu FLensburg, Germany

* These Authors CONTRIBUted EQUALly.

Corresponding author: Johanna Bendas, Department of Psychotherapy and Psychosomatics, Universitätsklinikum Carl Gustav Carus, Fetscherstraße 74, 01307 Dresden 


\section{Abstract}

Slow stroking touch activates C-tactile fibres in the human skin and is typically described as pleasant. Over a longer duration of stroking, affective habituation sets in, indicated by a reduction in rated pleasantness. However, it is not clear whether the group level effect is represented on an individual level. We analyze the stability of individual ratings of slow stroking touch and their relationship to behavioural and physiological measures.

48 participants ( 23 women) were repeatedly stroked with a velocity maximally activating C-tactile fibres. The perceived pleasantness was rated on a visual analogue scale. In order to examine the stability of pleasantness ratings, the experiment was repeated after a couple of days. During the experiment, electrocardiogram (ECG) and facial- electromyography (EMG) data were recorded.

On the group-level, previous results of affective habituation to touch were replicated and stable across sessions. On the individual level, however, less than half of the participants showed a significant reduction of pleasantness in the course of the experiment. Moreover, the remaining participants showed either no change, random rating behaviour or even an increase in pleasantness ratings during the course of the experiment.

The individual response patterns were variable across sessions but stable above the chance level. Furthermore, the response patterns could not be explicitly associated with any of the behavioural or physiological measures. Our findings indicate a lack of group-to-individual generalizability for affective habituation to touch. The variability of rating patterns over time indicates that they are not conclusively determined by stable individual characteristics. Future research investigating touch should favour a more individual approach to the more commonly applied group analysis.

\section{Keywords}

Affective habituation to touch, group-to-individual generalizability, C-tactile afferents 
Abbreviations

CT

C-tactile

ECG

electrocardiogram

EMG

electromyography

HRV

heart rate variability

RMSSD

root mean square of successive RR interval differences

SDNN

standard deviation of NN intervals 


\section{Introduction}

Affective touch describes the typically pleasant sensation of being touched and slowly stroked (Löken et al., 2009; McGlone et al., 2014). This specific kind of touch activates a specialized group of nerve fibres in the human hairy skin, the so-called C-tactile (CT) afferents (McGlone et al., 2014). These afferents react with highest firing rates to stroking stimulations performed with light pressure at a velocity of 1 to $10 \mathrm{~cm} / \mathrm{s}$ (Löken et al., 2009), and when the stroking is performed at skin temperature (Ackerley et al., 2014). CT afferents have been suggested to contribute to the emotional and affective value of interpersonal tactile interactions (Löken et al., 2009; Olausson et al., 2010).

Affective touch becomes less pleasant when it is repeated in the same way for a longer time, a phenomenon that has thus far been observed on a group level (Triscoli et al., 2014; Sailer et al., 2016b; Triscoli et al., 2017a). On average, humans initially evaluate repeated slow stroking touch applied to their forearm as pleasant. After a couple of minutes however, pleasantness ratings start to drop and after 40 to 50 minutes, the same stroking touch is experienced as neutral or slightly unpleasant, indicating a slow mechanism of "touch satiety" or "affective habituation to touch". These findings were based on group-level analyses and attributed to the general importance of touch for human interaction, bonding and well-being. While affective habituation to certain repeatedly presented stimuli is an effect that has been shown across sensory modalities such as the olfactory and auditory sense (e.g. Dijksterhuis and Smith, 2002; Mutschler et al., 2010; Ferdenzi et al., 2014), the specific mechanisms behind the phenomenon of affective habituation to touch mostly remain unclear. Generally speaking, habituation is a coping and learning mechanism of individuals that are surrounded by numerous stimuli of different sensory qualities in order to improve the responsiveness for new and potentially crucial inputs (Harris, 1943). The prolonged or repeated presentation of a certain stimulus typically leads to a decreased sensitivity to that stimulus (Dalton, 2000; Leventhal et al., 2007). Affective habituation as a subform of habituation describes the decrease of affective reaction with the repeated presentation of emotional stimuli (Leventhal et al., 2007). In previous experiments observing affective habituation in humans in the tactile, visual, olfactory and auditory sense (Mutschler et al., 2010; Ferdenzi et al., 2014; 
Triscoli et al., 2014; Mastria et al., 2017), the observed modulation of responsiveness towards the repeated stimuli was most commonly directed to more hedonically neutral values - so, for example, odours that were initially perceived as pleasant would be rated as less pleasant after repeated presentation, and odours initially evaluated as unpleasant would be perceived as less unpleasant in the course of the experiment. Both top-down and bottom-up processes may account for habituation. The effect of decreasing levels of perceived pleasantness of any repeatedly presented stimulus for example might be caused by a down-regulation of peripheral receptors resulting in effectively lower levels of perception. Likewise, depending on the stimulus presented, higher-order cerebral processing might modulate the organism's responsiveness for the specific stimulus in order to improve the availability and thus reaction towards more beneficial or potentially hazardous stimuli. In another experiment on affective habituation in C-tactile targeted touch, repeated tactile stimulation (Sailer et al., 2016b) resulted in decreased brain activations in the somatosensory areas (S1 and S2), suggesting a central habituation to the repeated stimulation (Leventhal et al., 2007). At the same time however, activation in reward areas such as the orbitofrontal gyrus (OFC) and putamen increased significantly with time. Such an increase in OFC activity is the opposite of what would be expected following reward devaluation (for example, due to satiety), where OFC activity typically decreases following consumption (e.g. Gottfried, 2003).

Affective habituation to touch or "touch satiety" probably reflects a combination of central and peripheral mechanisms. In addition, repeated touch stimulation might be evaluated differently by different individuals based on previous experiences with affective touch as well as personality traits. A major prerequisite for pinning down the mechanisms underlying affective habituation to touch is the possibility to generalize from grouped data to the individual level (Fisher et al., 2018, see also Adolf and Fried, 2019 for controversial discussion). Research in the broader social and medical sciences has historically relied on group level data analysis, assuming that the detected effects are scalable to the global population. However, individuals do not necessarily represent an average of the given population and for affective touch perception (but not habituation), it has been shown, that only about $40 \%$ of the 
individuals rating patterns align with the group response (Croy et al., 2020). In our present study, we aimed to examine the phenomenon of affective habituation to touch on both group as well as individual level in order to determine whether affective touch habituation is a general phenomenon, in individual trait or a state behaviour.

As the evaluation of a repeated hedonic stimulus might be influenced by individual differences in personality or behavioural traits (Hunter and Schellenberg, 2011; Ellingsen et al., 2016), the connection between affective habituation to touch and these characteristics seemed worth exploring. In addition to the subjective measure of hedonic evaluation of tactile stimuli, we aimed at complementing the representation of the reaction towards repeated stimuli with objective measures. Physiological responses may indicate varying degrees of emotional arousal (Cacioppo et al., 2000). Heart rate variability (HRV) is derived from an electrocardiogram and may indicate a participant's ability to regulate emotions (Appelhans and Luecken, 2006; Thayer et al., 2012; Holzman and Bridgett, 2017). Recent studies indicate that repeated CT-targeted touch stimulation may positively influence HRV (Triscoli et al., 2017a; Van Puyvelde et al., 2019a, 2019b). Another implicit measure for the hedonic evaluation of affective stimuli that has recently been applied in affective touch research and was included in this study design is facial electromyography (EMG) recordings of the corrugator and zygomaticus muscles (Pawling et al., 2017; Mayo et al., 2018; Ree et al., 2019).

In the present study, we aimed to replicate previous findings of affective habituation to touch on the group level and to follow up these previous findings with analyses on the individual level. We a) examined the percentage of individuals who show affective habituation to touch, previously described as a slow decrease in perceived pleasantness on a group-level (Triscoli et al., 2014; Sailer et al., 2016b; Triscoli et al., 2017a). We furthermore hypothesized that b) affective habituation to touch is a trait, hence that individuals reliably show such a pattern and c) that individual response patterns are related to physiological and behavioural data. For the latter hypothesis we investigated how affective habituation to touch relates to physiological data and constructs which have been shown to be related to general affective touch perception, namely EMG response (Mayo et al., 2018; Ree et al., 2019), heart 
rate variability (Triscoli et al., 2017b, 2017a) and to well-being (Field, 2002; McGlone et al., 2014) and reward sensitivity (Rolls, 2000; Sailer et al., 2016b). We hypothesized that participants with higher reward sensitivity and better subjective well-being would present a slower affective habituation to touch over time and that the effect of affective habituation to touch would be observable in facial EMG recordings as an implicit hedonic measure in a complementary way. In accordance with previous findings, we further hypothesized that repeated affective touch stimulation would have a positive effect on heart rate variability and show increasing levels with time.

\section{Experimental Procedures}

In total, 48 subjectively healthy participants (23 women, mean age $26.75 \pm 3.96$, range $21-38$ ) took part in the experiment. The study design was approved by the ethics committee of the Medical Faculty at the University of Dresden and the participants gave their informed consent prior to the investigations. All of the participants received a financial compensation of 20 Euro for their participation. Post hoc sensitivity analysis was performed using G*Power 3.1.9.2 and revealed that this sample size was sufficient to detect medium to large effects $[\mathrm{w}=0.41]$ of stability between session one and two $\left[\chi^{2}\right.$ test; $\mathrm{df}=1 ; \alpha=0.05 ; 1-\beta=0.8]$.

\section{Setup}

The study was part of a larger examination investigating physiological and subjective responses to repeated tactile and olfactory stimuli. The participants were seated in a comfortable chair in front of a computer screen with their left forearm placed on a soft cushion behind a curtain. Over the course of 30 minutes, the participants received repeated slow stroking touch applied by a trained experimenter seated behind said curtain. The participant rated the perceived pleasantness of the stimuli on a visual analogue scale on the computer screen. For olfactory stimulation, the odour Hedione (Firmenich, Meyrin, Switzerland) was presented repeatedly over the course of 15 minutes. The odorant was always presented after the completion of the slow stroking touch. During both conditions, facial EMG and heart rate were recorded - the electrodes were applied approximately 5-10 minutes prior to the start of the experiment. The examination was repeated in a second session (mean time between sessions $10.7 \pm 4.4$ 
days, range 6-20 days). The odour condition was applied in 36 participants and will not be covered in this publication. For more information on the odour condition and the results, we refer to our recent publication (Ree et al., 2020).

Between both sessions, the participants completed a set of questionnaires which contained the Temporal Experience of Pleasure Scale (TEPS, (Gard et al., 2006), which examines reward sensitivity, as well as the Diener Flourishing Scale (FS, (Esch et al., 2013) measuring subjective psychological wellbeing. The questionnaires further involved the Relationship Assessment Scale (RAS, (Sander and Böcker, 1993) measuring the satisfaction with the current relationship and partner and the Social Network Index (Cohen et al., 1997) sizing the quantity of social contacts as well as the diversity and structure of the social network. To investigate relations between the observed patterns of affective habituation to touch and other individual traits, the questionnaires included the Big Five Inventory (BFI, Lang et al., 2001) examining personality traits on the five different subcategories: openness to experience, conscientiousness, extraversion, agreeableness and neuroticism. The Patient Health Questionnaire (PHQ, (Spitzer et al., 1999) was applied as a screening method for mental health. All of the questionnaires were administered in German language. For each of the applied questionnaires, the total score or, if applicable, the scores for according sub-scales were calculated prior to the analysis.

In each session, the participants were stimulated on the left forearm with slow-stroking touch at a velocity of $3 \mathrm{~cm} / \mathrm{s}$ and a pressure of approximately $0,4 \mathrm{~N}$. Each trial consisted of a single-direction stroke in a proximal to distal direction across a distance of $10 \mathrm{~cm}$. The strokes were performed by a trained experimenter using the flat hand, guided by an auditory cue via headphones. The participants were asked to take a seat on a comfortable chair in front of a computer screen and place their left forearm on a pillow behind a curtain. Behind the curtain two experimenters were seated out of sight of the participant, so that the participant did not know which one of the experimenters performed the actual stroking. In each session, the participants received 60 CT-targeted stimulations (trials) with an intertrial-interval of approximately 25 seconds. After each trial, the participants rated the perceived pleasantness on a visual analogue scale which was given the anchor points "unpleasant" and "pleasant" 
and was presented on a computer screen. No further questions were asked in order to not distract participants and hence not to interfere with the affective habituation process as well as in accordance with previously conducted work (e.g. Sailer et al., 2016b). To take into account potential cases of touch aversion (Wilhelm et al., 2001; Kaiser et al., 2016; Strauss et al., 2019), a stop criterion was introduced: whenever a participant rated five consecutive tactile stimulations as "unpleasant" (ratings between 10 to -8$)$, the experiment was terminated immediately. This premature termination of the experiment was applied to one participant in the first session after 49 trials.

One participant did not attend session 2 due to illness. For the purpose of investigating the stability between session 1 and 2, the data of this participant in session 1 was not included in the analysis. Hence, the data of 47 participants were included in the analysis described here.

Data acquisition and processing

Facial electromyography (EMG) and electrocardiography (ECG) data were sampled at $1000 \mathrm{kHz}$ with the BioPac MP150 Nomadix wireless system (BioPac Systems Inc, Santa Barbara, CA, USA). Data were recorded using AcqKnowledge software (version 4.4, BioPac Systems Inc, Ca, USA). .

The EMG electrodes were positioned over the left corrugator supercilii and zygomaticus major muscles (Fridlund and Cacioppo, 1986; Tassinary et al., 2007). The reference electrode was placed on the participant's forehead. The second immediately preceding the onset of each tactile stimulus was designated as baseline and the 3.3 seconds when the touch was applied were designated as the activity period. E-prime (Psychology Software Tools, Pittsburgh, PA) controlled the timing of stimulus onset (stroking and odours) by sending an auditory cue to the experimenter. E-prime also marked this stimulus onset in the EMG/ECG recordings and controlled the timing of VAS-scale presentation.

The heart rate data were collected using a three lead set-up with surface electrodes placed inferior to the right and left clavicle and left $8^{\text {th }}$ rib, as described by BioPac (AcqKnowledge 4.4). A 3-minute baseline was collected prior to the experiment. In a visual inspection, artifacts and multiple peaks were crossreferenced to the raw ECG file and manually adjusted as appropriate. Every trial contained the stroking stimulation of the forearm $(3.3 \mathrm{sec})$ and the following pleasantness rating period. To remove outliers 
from the facial EMG data, the residuals were categorized into standard deviation units (i.e. with a distribution centered around a mean of 0 and a standard deviation of 1 ). We defined values outside the \pm 2.58 standard deviations as outliers and these trials were excluded from the analysis (Field, 2013). In total, roughly $5 \%$ of the facial EMG trials were affected from this data selection.

The ECG data were split into time bins of three minutes and visually inspected for normality on a participant-by-participant basis, before the measures of interest were calculated. These measurements were the standard deviation of NN intervals (SDNN) and the root mean square of successive RR interval differences (RMSSD) (Shaffer and Ginsberg 2017). The RMSSD reflects the beat-to-beat variance in heart rate and is used to estimate changes in HRV induced by a higher vagal activity. The SDNN is a broader measure of factors contributing to fluctuations of the HRV (Shaffer and Ginsberg, 2017). Artifacts and missing beats were cross-referenced to original raw data file and corrected according to recommendations set out by international recommendations (Task Force of the European Society of Cardiology and the North American Society of Pacing and Electrophysiology, 1996). The ECG data were further analysed in Kubios HRV Standard 3.1.0 [Kubios Oy, Kuopio, Finland] (Tarvainen et al., 2009). Due to technical problems, six tachograms could not be analysed (four from session 1 and two from session 2) and were consequently removed from the statistical analysis. Three participants were pregnant during the time of testing. As pregnancy has been reported to affect HRV measures (e.g. (Stein et al., 1999), these data were scrutinized. One participant had HRV values outside the mean $\pm 2 \times S D$, and these were not included in the analysis.

Thus, 43 participants were included in the HRV analysis for session 1, and 44 participants were included in session 2 (one participant cancelled the second session due to illness). The facial EMG and pleasantness analyses were based on responses from 48 participants and 47 participants, for session 1 , and session 2, respectively.

\section{Data clustering and analysis}

Data were analysed in SPSS Statistics 25.0 for Windows (IBM Corp, Armonk, NY, USA). We analysed the rating patterns on the group level separately for each session with a repeated measure ANOVA with the 
independent variable "trial" (60) and the dependent variable "pleasantness" and - following previous results on affective touch satiety and habituation (Triscoli et al., 2014) - we aimed for the linear effect of "trial" on "pleasantness". We first tried a clustering according to a previously used approach (Sailer et al., 2016a) which fitted the ratings of each person and condition to a linear, exponential and two-step model. The two-step model assumed a constant pattern with a sudden shift to a lower or higher level at a particular trial. The model with the smallest Akaike Information Criterion was assumed to be the best model, and was further categorised according to the direction of the change as positive, fast negative or slow negative. A fast negative change was defined as one where $80 \%$ of the total change (difference between initial and final value estimated by the best fitting model) occurred prior to trial 29 (touch condition), otherwise the negative change was classified as "slow". This way of classification led to 9 categories of rating patterns.

However, these categories left us with too few cases for further subsequent analyses in some of the categories. We thereafter decided to simplify this approach to the basic patterns according to our hypotheses: increase, decrease or constant rating behaviour. This exploratory approach was applied post hoc. In order to analyse the individual patterns of ratings, we performed a linear regression analysis for each individual with the independent variable "trial" and the dependent variable "pleasantness" separately for each session. A linear model was chosen in accordance to the previous study by Triscoli and colleagues (Triscoli et al., 2014). Level of significance was set to $p<0.05$, two-sided. Depending on whether a significant linear rating pattern was observed, participants were grouped into three categories: a) significant linear decrease of ratings over time (decrease), b) significant linear increase of ratings over time (increase) and c) no significant linear fit (unspecified).

Participants with rating curves in category "a" showed on average a $40 \%$ decrease of ratings over the trials. Visual inspection of the individual ratings suggested that there were two further underlying categories: participants who showed a steep decrease at the beginning and those who had a continuous decrease. We therefore further divided this group: The participants showing the majority of the decrease (namely 75\%) already in the first 6 trials were categorized as "fast decreasers"; the remainder 
of the participants in this group were categorized as "slow decreasers" (compare Figure 1). The categorization was based on visual inspection and performed in order to allow for a more fine-tuned retest analysis.

The "unspecified" category comprised participants who rated the pleasantness of the repeated tactile stimuli as nearly constant throughout the experiment and participants that appeared to apply a certain randomness in their ratings with many ups and downs, and thus a high variance. For further description, all participants whose data had no significant linear fit and who had a low variance of ratings over time (mean square of the residuals $<1$ ) were categorized into the "constant" group. Participants whose ratings did not follow a significant linear fit and whose mean square residuals exceeded 1 were categorized into the "random" group (compare Figure 1). This split-up resulted in five different groups for the analysis: "fast decreaser", "slow decreaser", "increase", "random" and "constant".

The number of participants categorized into each group was counted for session 1 and session 2, as was the number of individuals who remained in the same group in both sessions. In order to test the significance of the stability, we performed a binominal test against the chance level of $20 \%$.

The observed and predicted likelihood of the stability of categories between sessions was calculated as follows: Predicted likelihood was calculated based on the data from session 1. According to our data for example, $17 \%$ of the participants of session 1 presented a fast decrease of pleasantness ratings. In order to calculate the predicted likelihood of presenting a fast decrease in both sessions, this percentage was squared $(0.17 * 0.17=0.029(2.9 \%))$. The observed likelihood of stability across sessions was calculated as the percentage of participants within a designated rating category in session 1 that were assigned to the same category in session 2. For example, exactly half of the participants whose ratings decreased fast in the first session could be described by the same pattern in session 2. The observed likelihood here equals $0.17 * 0.5=0.085(8.5 \%)$.

In order to evaluate whether different patterns of affective habituation to touch were related to physiological changes, we compared the dependent variables of mean heart rate variability (SDNN, 
RMSSD), heart rate (RR) and mean EMG changes (m. zygomaticus and m. corrugator) for different rating patterns using separate generalized linear mixed models. In order to smooth physiological data, the data was split up into 6 bins, each comprising the mean of 10 subsequent stroke trials. Each subject was included as random slope and bins served as repeated measurement. Rating patterns (factor levels constant, random, increase, decrease slow, decrease fast) and bins (factor levels from 1 to 10) were included as fixed factors. However, due to the small cell occupancy (e.g. $n=5$ for "constant"), we categorized only two groups of rating patterns in accordance with the original hypothesis (slow/fast decrease vs. all other patterns).

For more detailed information on the physiological data we refer to another publication where the course of EMG and ECG data throughout the experiment is presented both graphically and statistically analyzed (see Ree et al., 2020). In the present study, we focused on the individual dynamics of affective habituation to touch and its relation to physiological and behavioral data.

In order to evaluate whether different patterns of affective habituation to touch were related to state or trait effects, we compared the dependent variables of PHQ, BFI, TEPS, RAS and FS to rating patterns using separate multivariate ANOVAs. Rating patterns served as fixed factors. Again, due to the small cell occupancy, we categorized only two groups of rating patterns in accordance with the original hypothesis (slow/fast decreaser vs. all other patterns)

For reasons of transparency, all mean values of the analysis concerning the physiological and questionnaire data are additionally presented for all rating patterns in the supplementary table.

\section{Results}

Dynamics of affective habituation to touch on the group-level

On a group level, pleasantness ratings significantly dropped in the course of the experiment in session 1 as well as session 2, and the decrease followed a linear pattern (ANOVA results - session 1: $F[1,46]=9.2$, $p=0.004, \eta^{2}=0.166$; session 2: $F[1,46]=8.52, p=0.005, \eta^{2}=0.156$; compare figure 1$)$. Mean pleasantness ratings in session 1 dropped from 4,93 (first stimulation, $\mathrm{N}=48$, maximum 10 , minimum -2.67) to 3.77 (last stimulation, $\mathrm{N}=47$, maximum 10 , minimum -2.67 ). In session 2 , from the first to the last tactile 
stimulation the mean perceived pleasantness dropped from 4,68 ( $N=47$, maximum 10 , minimum -1.33) to $3.53(\mathrm{~N}=47$, maximum 10 , minimum -5.33$)$. While the perceived pleasantness of the stroking stimulations in the course of the experiment decreased, pleasantness ratings at the very end of the experiment still remained positive on average, taking into account the anchor points of the applied visual analogue scale from -10 to 10 .

Individual dynamics of affective habituation to touch

The distribution of the categories of rating behaviour in session 1 is displayed in Figure 1B and can be described as follows: 20 participants (42.6\%) showed a decrease in individual ratings that was similar to the decrease of the group average: of those 20, 8 participants had a fast decrease and 12 a slow decrease of pleasantness ratings. Six participants (12.8\%), however, showed an opposite rating pattern and rated the tactile stimulation as more pleasant at the end of the experiment. Five participants (10.6\%) presented a constant rating behaviour and $34 \%$ of the participants, namely 16 , showed a random rating behaviour, implying that no significant linear fit at $p<0.05$ was observed for the trial-dependent rating patterns of those participants. The distribution of participants per group was fairly similar in session 2 . However, individuals changed categories (Table 1). 


\section{A Averaged rating patterns per category}

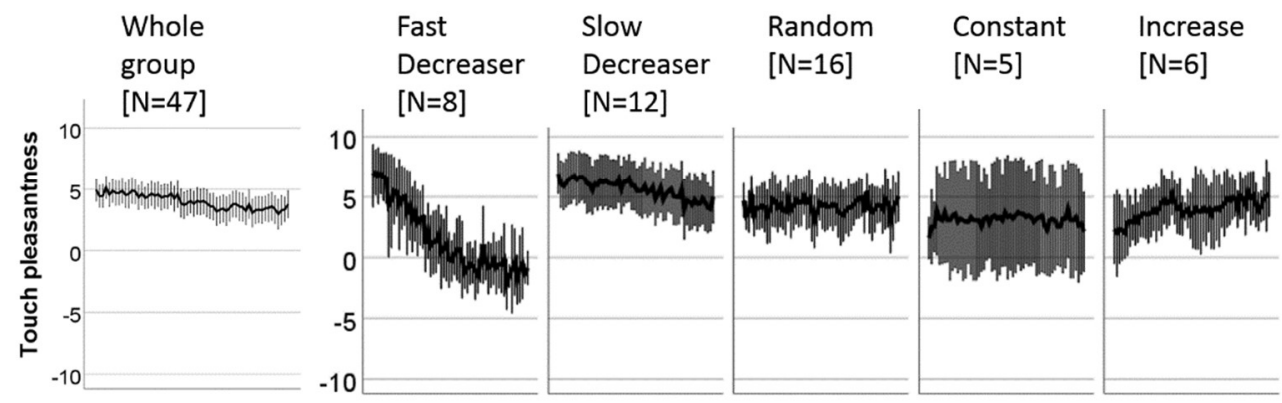

\section{B Individual rating patterns per category}
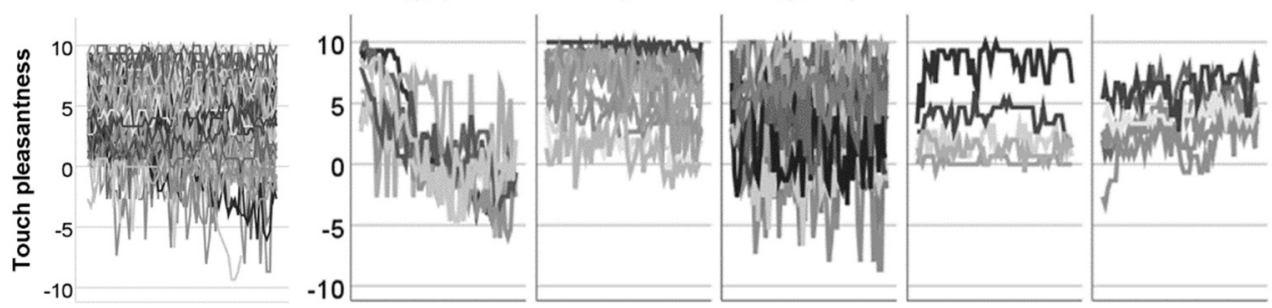

Figure 1 Pleasantness ratings during the course of session 1. A) Averaged across all participants, the pleasantness ratings decreased during the course of the experiment. Divided into the 5 different groups, the participants presented varying rating behaviour with the expected decrease of perceived pleasantness as well as increase, constant and random rating behaviour. B) Individual pleasantness ratings of all participants per group.

Stability of affective habituation to touch patterns on an individual level The participants' responses were investigated with regard to their stability or change of categories between session 1 and session 2. Out of the 8 participants who were classified as fast decreasers in session $1,50 \%(n=4)$ could be assigned to the same category in session 2 . The other participants who originated from the category of fast decreaser in session 1 were mainly categorized as slow decreasers $(n=3)$ in session 2 or - to a lesser extent - as random raters ( $n=1$; compare Table 1$)$.

For the slow decreasers, the constant and the increase group, the majority of participants remained in the same group during both sessions. Changes of categories were more frequent between similar categories (e.g. from increase to constant) and less between opposite categories (e.g. from increase to fast or slow decreaser; compare Table 1). An exception was the random category: all the participants with this rating behaviour were grouped into different categories in session 2. Out of the participants 
that showed a decrease (slow or fast) in pleasantness ratings in session 1, $70 \%$ (14 out of 20) presented a decreasing rating pattern in the second session. Out of the participants that rated the repeated tactile stimulation as increasingly pleasant in the first session, none showed a decrease in pleasantness ratings in the second session. These participants were divided into the categories increase as well as constant and random in session 2.

In total, 17 (36\%) of the participants showed a stable rating pattern between session 1 and session 2 . This value exceeds the chance level of $20 \%$, given a mere coincidental distribution of the rating categories in session $2\left(\chi^{2}=4.03 ; p<0.001\right)$. The predicted and observed likelihood of stability of rating categories over sessions can be described as follows: For the category of fast decreaser, the predicted likelihood of stability of $2,9 \%$ was overruled by the observed likelihood of $8,5 \%$, which can be interpreted as the rating pattern of fast decreasers being more likely to be stable across sessions than predicted. A similar distribution in favor of the observed likelihood of stability was detected for the categories slow decreaser (predicted 6,5\%, observed 11,6\%), constant (predicted 1,1\%, observed $4,2 \%$ ) as well as increase. For the random category however, the observed likelihood of $8,5 \%$ was lower than the predicted likelihood of $11,6 \%$.

Patterns of affective habituation to touch and physiological and behavioral data There was no significant interaction between rating patterns and any of the physiological variables. However, we observed a significant main effect of rating patterns for heart rate $(F[1,168]=10.9$, $p=0.001$ ) with decreasers having a lower HR than persons with other rating patterns, and a significant main effect for HRV with decreasers having a higher SDNN than persons with other ratings patterns $(F[1,179]=5.4, p=0.022)$. For other variables, this effect was not significant.

There was further no significant relation between rating patterns and any of the questionnaire data $\left(\eta^{2}\right.$ $=0.00$ to 0.074$)$. The highest variance of rating patterns was explained by the BFI subscale "openness for new experiences $\left(\eta^{2}=0.074 ; p=0.085\right)$, which was the only variable with a significance below $p=0.01$. Decreasers reported a higher openness for new experience than individuals with any other rating pattern (compare supplementary table). 


\begin{tabular}{|c|c|c|c|c|c|c|c|}
\hline & $\begin{array}{c}\text { Rating category } \\
\text { Session } 2\end{array}$ & $\begin{array}{c}\text { Decrease } \\
\text { fast }\end{array}$ & $\begin{array}{c}\text { Decrease } \\
\text { slow }\end{array}$ & Random & Constant & Increase & Total \\
\hline \multicolumn{8}{|l|}{ Rating category } \\
\hline Session 1 & & & & & & & \\
\hline Decrease fast & & 4 & 3 & 1 & - & - & 8 \\
\hline Decrease slow & & 2 & 5 & 2 & 2 & 1 & 12 \\
\hline Random & & 1 & 5 & 4 & 1 & 5 & 16 \\
\hline Constant & & - & 1 & 1 & 2 & 1 & 5 \\
\hline Increase & & - & - & 2 & 2 & 2 & 6 \\
\hline Total & & 7 & 14 & 10 & 7 & 9 & 47 \\
\hline
\end{tabular}

Table 1 Quantity of participants per rating category in session 1 and 2. The distribution of participants across the 5 rating categories was similar in session 1 as well as session 2. The bold numbers represent the number of participants that were grouped in the same category in both sessions.

\section{Discussion}

In the present study, a lack of group to individual generalizability was documented in the domain of affective habituation to touch. The hedonic rating behaviour observed on the group level during repeated tactile stimulation was representative for less than half of the participants when analysed on an individual level.

Rather than the group average, the individual data could better be described by five different categories of rating patterns, and 31 out of 47 participants could be matched to empirically derived categories of hedonic rating behaviour. This is in line with a previous study showing different patterns of dynamic hedonic evaluation in an experiment using repeated presentation of odours (Sailer et al., 2016a). Here, the perceived pleasantness of the stimuli during the course of the experiment increased for some of the participants as well. 
Rating patterns on an individual level were stable above chance across sessions and post hoc sensitivity analysis showed that our sample size allowed statistical confirmation of a medium to large effect, while smaller effects are not covered. The vast majority of participants that showed a decrease in pleasantness ratings in the first session demonstrated decreasing pleasantness ratings in session 2 as well. None of the participants initially presenting an increase in session 1 changed to a decrease in pleasantness ratings in the second session. This suggests decreasing as well as increasing hedonic rating patterns to be more persistent and potentially trait-related.

Within the different categories the observed likelihood of pattern stability between session 1 and 2 was slightly higher than the predicted likelihood for all of the categories - except for random ratings.

The category of random rating behaviour seems particularly striking: This category gathers all the participants whose dynamic hedonic rating behaviour did not significantly relate to the design of the study. In session 1, almost a third of the participants were best described by this random category, so the question arises whether these participants were sincerely indifferent to the applied affective touch due to varying neurophysiological properties or simply boredom. Individuals might differ in their fatigue of nerve fibers on peripheral or central level which might result in such cases of hedonic indifference. Future studies investigating the dynamics of hedonic evaluation could take this into account and report such a number of potentially "indifferent" participants in order to improve the quality of group-level analysis and statements to be drawn from it. Another important approach for future research that might shed more light on the presented results would be the replication of the presented rating categories in a, potentially larger, independent sample.

Based on the group-averaged result, it is tempting to state that affective habituation to touch is a stable behaviour, but the individual results seem to tell a different story. In the literature, the concept of affect habituation is described as a two-factor model: with repeated exposure to affective stimuli, the familiarity of the stimulus increases which might facilitate the processing of that stimulus and hence increase the perceived pleasantness (Leventhal et al., 2007). With increasing familiarity of the stimulus however, a certain tiredness of the stimulus appears and results in the described affect habituation 
effect. Our data suggests that these two different factors might be weighed differently in the participating individuals. Specifically, while some of them showed the expected pattern of decreasing pleasantness ratings during the course of the experiment, others reported an increased pleasantness with time. This increase in perceived pleasantness throughout the experience might suggest a centrally modulated affective habituation effect. Individual experiences with affective touch and higher-order processes might improve the responsiveness towards affective touch with repeated presentation as it is interpreted as an evolutionarily important and potentially beneficial stimulus for the initiation and maintenance of interpersonal relationships. These increasing levels of perceived pleasantness of affective touch with repeated stimulation are not represented on the group-level and, to our knowledge, this is the first presentation of such data in affective touch. The lack of test-retest-reliability of the presented rating patterns on the individual level did not support the hypothesis of affective habituation to touch being a stable trait.

The physiological and behavioural data drawn from ECG, facial EMG and questionnaires did also not reveal any traits connected to the occurrence of these affective habituation to touch patterns. This might be explained by methodological issues or due to the rather small sample size of the study which results in a lack of power for the detection of weak effects. The only personality trait linked to a higher likelihood of decreasing perception of pleasantness during the experiment was openness for experiences. Participants scoring higher on this subscale tend to be sensation-seekers and indeed perceive the repeated tactile stimulus as less salient or interesting and thus less pleasant. This is in line with previous research on liking of music as a repeated auditory stimulus in humans where, likewise, higher levels of openness for experiences could be connected to a decrease in liking as a function to the exposure to the same music (Hunter and Schellenberg, 2011). The ECG data revealed participants with decreasing pleasantness during the experiment to have a higher heart rate variability. This finding is in line with previous reports of repeated CT-targeted touch being connected to higher levels of heart rate variability (Triscoli et al., 2017b). The coherence between decreasing perception of pleasantness and heart rate variability might also indicate higher levels of attention towards hedonic stimuli in 
participants, reflecting a greater capacity for emotion regulation (Appelhans and Luecken, 2006; Thayer et al., 2012; Holzman and Bridgett, 2017). However, we were unable to demonstrate a coherence between the RMSSD and pleasantness ratings. As the RMSSD is recommended for shorter time measurements (Force, 1996), the relationship between HRV and individual pleasantness ratings needs to be interpreted cautiously.

Properties of affective touch have been studied broadly on group level; however, it remains unclear which individual factors might contribute to those relations. Affective touch in everyday-life is more versatile and diverse than as examined under standardized laboratory conditions and most challenging to be studied independently from covarying factors on a group-level. The rather high variability of individual dynamic pleasantness ratings in our data might thus be explained by contextual or underlying factors such as mood or boredom, which were not determined in our study, or simply a general randomness in individual ratings. Effects of mood, anxiety or boredom might also not only relate to the individual rating behaviour within the experiment (Ellingsen et al., 2016), but also explain differences in the dynamics of perceived pleasantness between sessions, e.g. participants being in a better or worse, more open or more anxious mood in session 1 compared to session 2. Future studies on affective habituation to touch should take these factors into account.

A clear limitation of the study design is the lack of a tactile control condition in the experimental set-up, which limits the ability to draw conclusions solely on CT afferents. The affective touch stimulation applied in this study design was demonstrably aiming at optimally activating CT afferents. However, the same stroke applied on hairy skin will always stimulate different other afferent fibers as well, such as Abeta fibers, and the sense of touch is a complex construct with many factors contributing to the sensation perceived by the touched individual. The presented results might thus represent the effects of any repeatedly applied hedonic tactile stimulus. Another limitation worth mentioning is the lack of examining the participants' perception of intensity of the repeated tactile stimulus in the course of the experiment. A peripherally driven affective habituation towards the touch stimulation might be detectable with decreasing levels of perceived intensity analogous to decreasing levels of perceived 
pleasantness. However, the study design considered the necessary interstimulus intervals in order to avoid a presentation of following stimulus during a potential refractory phase of the previous stimulus.

Our data suggest that affective touch appreciation is represented differently on an individual level compared to the group level. Observation and description of individual data demonstrate that individual evaluation of slow stroking touch differs from the group average and thus, assumptions based on grouplevel data need to be made carefully.

\section{Acknowledgements}

The study was supported by the Research Council of Norway, project number: 267446/F10, and the German Academic Exchange Service (DAAD), project number: 0945159. 


\section{References}

Ackerley R, Wasling HB, Liljencrantz J, Olausson H, Johnson RD, Wessberg J (2014) Human C-Tactile Afferents Are Tuned to the Temperature of a Skin-Stroking Caress. J Neurosci 34:2879-2883.

Adolf JK, Fried El (2019) Ergodicity is sufficient but not necessary for group-to-individual generalizability. Proc Natl Acad Sci 116:6540-6541.

Appelhans BM, Luecken $L J$ (2006) Heart rate variability as an index of regulated emotional responding. Rev Gen Psychol 10:229-240.

Cacioppo JT, Berntson GG, Larsen JT, Poehlmann KM, Ito TA (2000) The psychophysiology of emotion. Handb Emot 2:173-191.

Cohen S, Doyle WJ, Skoner DP, Rabin BS, Gwaltney JM (1997) Social ties and susceptibility to the common cold. Jama 277:1940-1944.

Croy I, Bierling A, Sailer U, Ackerley R (2020) Individual Variability of Pleasantness Ratings to Stroking Touch Over Different Velocities. Neuroscience:S0306452220301895.

Dalton P (2000) Psychophysical and Behavioral Characteristics of Olfactory Adaptation. Chem Senses 25:487-492.

Dijksterhuis A, Smith PK (2002) Affective habituation: Subliminal exposure to extreme stimuli decreases their extremity. Emotion 2:203-214.

Ellingsen D-M, Leknes S, Løseth G, Wessberg J, Olausson H (2016) The Neurobiology Shaping Affective Touch: Expectation, Motivation, and Meaning in the Multisensory Context. Front Psychol 6 Available at: http://journal.frontiersin.org/Article/10.3389/fpsyg.2015.01986/abstract [Accessed August 21, 2020].

Esch T, Jose G, Gimpel C, von Scheidt C, Michalsen A (2013) Die Flourishing Scale (FS) von Diener et al. liegt jetzt in einer autorisierten deutschen Fassung (FS-D) vor: Einsatz bei einer Mind-Bodymedizinischen Fragestellung. Complement Med Res 20:267-275.

Ferdenzi C, Poncelet J, Rouby C, Bensafi M (2014) Repeated exposure to odors induces affective habituation of perception and sniffing. Front Behav Neurosci 8 Available at: http://journal.frontiersin.org/article/10.3389/fnbeh.2014.00119/abstract [Accessed August $18,2020]$.

Field A (2013) Discovering statistics using IBM SPSS statistics. sage.

Field T (2002) Infants' need for touch. Hum Dev 45:100-103.

Fisher AJ, Medaglia JD, Jeronimus BF (2018) Lack of group-to-individual generalizability is a threat to human subjects research. Proc Natl Acad Sci 115:E6106-E6115.

Force T (1996) Standards of measurement, physiological interpretation and clinical use. Task force of the European Society of Cardiology and the North American Society of Pacing and Electrophysiology. Circulation 93:1043-1065.

Fridlund AJ, Cacioppo JT (1986) Guidelines for human electromyographic research. Psychophysiology 23:567-589. 
Gard DE, Gard MG, Kring AM, John OP (2006) Anticipatory and consummatory components of the experience of pleasure: a scale development study. J Res Personal 40:1086-1102.

Gottfried JA (2003) Encoding Predictive Reward Value in Human Amygdala and Orbitofrontal Cortex. Science 301:1104-1107.

Harris JD (1943) Habituatory response decrement in the intact organism. Psychol Bull 40:385-422.

Holzman JB, Bridgett DJ (2017) Heart rate variability indices as bio-markers of top-down selfregulatory mechanisms: A meta-analytic review. Neurosci Biobehav Rev 74:233-255.

Hunter PG, Schellenberg EG (2011) Interactive effects of personality and frequency of exposure on liking for music. Personal Individ Differ 50:175-179.

Kaiser MD, Yang DY-J, Voos AC, Bennett RH, Gordon I, Pretzsch C, Beam D, Keifer C, Eilbott J, McGlone F, Pelphrey KA (2016) Brain Mechanisms for Processing Affective (and Nonaffective) Touch Are Atypical in Autism. Cereb Cortex 26:2705-2714.

Leventhal AM, Martin RL, Seals RW, Tapia E, Rehm LP (2007) Investigating the dynamics of affect: Psychological mechanisms of affective habituation to pleasurable stimuli. Motiv Emot 31:145157.

Löken LS, Wessberg J, McGlone F, Olausson H (2009) Coding of pleasant touch by unmyelinated afferents in humans. Nat Neurosci 12:547.

Mastria S, Ferrari V, Codispoti M (2017) Emotional Picture Perception: Repetition Effects in FreeViewing and during an Explicit Categorization Task. Front Psychol 8:1001.

Mayo LM, Lindé J, Olausson H, Heilig M (2018) Putting a good face on touch: Facial expression reflects the affective valence of caress-like touch across modalities. Biol Psychol 137:83-90.

McGlone F, Wessberg J, Olausson H (2014) Discriminative and Affective Touch: Sensing and Feeling. Neuron 82:737-755.

Mutschler I, Wieckhorst B, Speck O, Schulze-Bonhage A, Hennig J, Seifritz E, Ball T (2010) Time Scales of Auditory Habituation in the Amygdala and Cerebral Cortex. Cereb Cortex 20:2531-2539.

Olausson H, Wessberg J, Morrison I, McGlone F, Vallbo $\AA$ (2010) The neurophysiology of unmyelinated tactile afferents. Neurosci Biobehav Rev 34:185-191.

Pawling R, Cannon PR, McGlone FP, Walker SC (2017) C-tactile afferent stimulating touch carries a positive affective value Tremblay F, ed. PLOS ONE 12:e0173457.

Ree A, Bendas J, Pabel L, Croy I, Sailer U (2020) Right between the eyes: Corrugator muscle activity tracks the changing pleasantness of repeated slow stroking touch. Physiol Behav 222:112903.

Ree A, Mayo LM, Leknes S, Sailer U (2019) Touch targeting C-tactile afferent fibers has a unique physiological pattern: A combined electrodermal and facial electromyography study. Biol Psychol 140:55-63.

Rolls ET (2000) The Orbitofrontal Cortex and Reward. Cereb Cortex 10:284-294.

Sailer U, Triscoli C, Croy I (2016a) Still Eating Despite Decreased Olfactory Pleasure-The Influence of Odor Liking and Wanting on Food Intake. Chem Senses 41:497-504. 
Sailer U, Triscoli C, Häggblad G, Hamilton P, Olausson H, Croy I (2016b) Temporal dynamics of brain activation during 40 minutes of pleasant touch. Neurolmage 139:360-367.

Sander J, Böcker S (1993) The German version of the Relationship Assessment Scale (RAS): A short scale for measuring satisfaction in a dyadic relationship. Diagnostica 39:55-62.

Shaffer F, Ginsberg JP (2017) An Overview of Heart Rate Variability Metrics and Norms. Front Public Health 5:258.

Spitzer RL, Kroenke K, Williams JB, Patient Health Questionnaire Primary Care Study Group (1999) Validation and utility of a self-report version of PRIME-MD: the PHQ primary care study. Jama 282:1737-1744

Stein PK, Hagley MT, Cole PL, Domitrovich PP, Kleiger RE, Rottman JN (1999) Changes in 24-hour heart rate variability during normal pregnancy. Am J Obstet Gynecol 180:978-985.

Strauss T, Rottstädt F, Sailer U, Schellong J, Hamilton JP, Raue C, Weidner K, Croy I (2019) Touch aversion in patients with interpersonal traumatization. Depress Anxiety.

Tarvainen MP, Niskanen J-P, Lipponen J, Ranta-Aho P, Karjalainen P (2009) Kubios HRV-a software for advanced heart rate variability analysis. In, pp 1022-1025. Springer.

Tassinary LG, Cacioppo JT, Vanman EJ (2007) The skeletomotor system: Surface electromyography.

Thayer JF, Åhs F, Fredrikson M, Sollers III JJ, Wager TD (2012) A meta-analysis of heart rate variability and neuroimaging studies: implications for heart rate variability as a marker of stress and health. Neurosci Biobehav Rev 36:747-756.

Triscoli C, Ackerley R, Sailer U (2014) Touch Satiety: Differential Effects of Stroking Velocity on Liking and Wanting Touch Over Repetitions. PLOS ONE 9:e113425.

Triscoli C, Croy I, Olausson H, Sailer U (2017a) Touch between romantic partners: Being stroked is more pleasant than stroking and decelerates heart rate. Physiol Behav 177:169-175.

Triscoli C, Croy I, Steudte-Schmiedgen S, Olausson H, Sailer U (2017b) Heart rate variability is enhanced by long-lasting pleasant touch at CT-optimized velocity. Biol Psychol 128:71-81.

Van Puyvelde M, Collette L, Gorissen A-S, Pattyn N, McGlone F (2019a) Infants Autonomic CardioRespiratory Responses to Nurturing Stroking Touch Delivered by the Mother or the Father. Front Physiol 10:1117.

Van Puyvelde M, Gorissen A-S, Pattyn N, McGlone F (2019b) Does touch matter? The impact of stroking versus non-stroking maternal touch on cardio-respiratory processes in mothers and infants. Physiol Behav 207:55-63.

Wilhelm FH, Kochar AS, Roth WT, Gross JJ (2001) Social anxiety and response to touch: incongruence between self-evaluative and physiological reactions. Biol Psychol 58:181-202. 